\title{
Resveratrol inhibits proliferation and promotes apoptosis via the androgen receptor splicing variant 7 and PI3K/AKT signaling pathway in LNCaP prostate cancer cells
}

\author{
MUSHI YE* , HUANSHU TIAN* ${ }^{*}$ SHANHONG LIN, JIERONG MO, ZHUO LI, XIAOJUN CHEN and JIANJUN LIU \\ Laboratory of Urology, Affiliated Hospital of Guangdong Medical University, Zhanjiang, Guangdong 524000, P.R. China
}

Received January 6, 2020; Accepted July 17, 2020

DOI: $10.3892 / \mathrm{ol} .2020 .12032$

\begin{abstract}
Prostate cancer is a common malignant tumor of the male genitourinary system and its incidence increases with age. Studies have shown that resveratrol (Res) inhibits cancer cell proliferation, migration, invasion and promotes apoptosis. The present study evaluated the effect of Res in two human prostate cancer cell lines (the androgen-dependent LNCaP cell line and the non-androgen-independent LNCaP-B cell line) on proliferation and apoptosis. A proliferation assay was used to demonstrate that Res inhibited proliferation of $\mathrm{LNCaP}$ and LNCaP-B cells in the range of 25-100 $\mu \mathrm{M}$, and the effect was time- and dose-dependent. Using flow cytometry, it was reported that various concentrations of Res induced apoptosis in LNCaP and LNCaP-B cells, and that the apoptotic effect of Res was dose-dependent. A chemiluminescence assay showed that Res inhibited prostate specific antigen levels in LNCaP and LNCaP-B cells. Reverse transcription quantitative-PCR showed that Res inhibited the expression of androgen receptor $(A R)$ in LNCaP and LNCaP-B cells at the mRNA level. Western blot analysis showed that Res suppressed the expression of AR protein as well as protein kinase B (AKT) phosphorylation. To study the effect of Res on the expression of AR splicing variant 7 (ARV7) and the PI3K/AKT signaling pathway in prostate cancer cells, as well as the underlying molecular mechanisms, the recombinant ARV7 expression vector Pcdna3.1-ARV7 was transfected into LNCaP and LNCaP cells and the aforementioned experiments were repeated. It was revealed that Res acted via the ARV7 and the AKT pathways. Taken together, the present results suggested that Res suppresses the proliferation of prostate cancer cells, promotes apoptosis and inhibits the expression of AR mRNA and protein. These effects likely
\end{abstract}

Correspondence to: Professor Jianjun Liu, Laboratory of Urology, Affiliated Hospital of Guangdong Medical University, 57 Renmin Road, Zhanjiang, Guangdong 524000, P.R. China

E-mail: jianjunliulab@163.com

*Contributed equally

Key words: prostate cancer, resveratrol, AR splicing variant 7, AKT signaling pathway, apoptosis resulted from inhibition of ARV7 and the AKT signaling pathway.

\section{Introduction}

Prostate cancer $(\mathrm{PCa})$ is a common malignant tumor in the male genitourinary system. According to estimates by American Cancer Society, in the United States, 164,690 new cases of PCa were diagnosed and PCa accounted for nearly $19 \%$ of all male newly diagnosed cancers in 2018 (1). Surgery, endocrine therapy, radiation and chemotherapy are the current preferred therapeutic approaches (2). For some patients with advanced $\mathrm{PCa}$, endocrine therapy is the treatment of choice (3), and this delays disease progression temporarily. Nevertheless, eventually PCa treatment becomes difficult due to the development of castration-resistant prostate cancer (CRPC) $(4,5)$.

The androgen receptor (AR) is considered to be the most important factor in the progression of PCa in CRPC (6,7). AR is a member of the nuclear receptor superfamily of proteins and is a class of nuclear transcription factors that facilitate entry into cells of testosterone and double-hydrogen testosterone to form androgen-androgen receptor complexes (8). AR and heat shock protein (HSP90) dissociate and are transported to the prostate, seminal vesicle and epididymis the nuclei of target tissues, such as the skeletal muscle system, which modulates activation and inhibition of target genes, thereby mediating the effects of androgens $(9,10)$. Studies have shown that abnormal expression of AR is one of the key factors in the progression of CRPC (11-13). Prostate specific antigen (PSA) has been suggested as a molecular target for prostate cancer because it is not only active in prostate tissues, but also plays a key role in the signaling pathway of prostate cancer (14). The combination of androgens and AR induces the dissociation of AR from HSP90 and stimulates AR phosphorylation to activate the activity of downstream proteins (15). These proteins activate or inhibit target genes, which regulate the proliferation, survival and production of PSA in prostate cells (16).

ARV7 is a truncated AR that functions as an AR shear-variant (AR-V). Alike AR, ARV7 retains an $\mathrm{NH}_{2}$-terminal domain (NTD) and DNA-binding domain (DBD), but lacks the full-length ligand-binding domain (LBD) of AR. The binding of androgen to AR LBD allows the ligand-binding receptor to enter the nucleus and then transcriptionally regulate androgen-responsive genes. Therefore, 
ARV7 is not available to bind to the androgen , and regulates the transcriptional activation of downstream target genes in the absence of androgens $(17,18)$. ARV7 is considered to play an important role in the process of castration resistance in PCa invasion, metastasis, hormone therapy resistance and biochemical recurrence (18).

Resveratrol (Res) is a natural polyphenol compound containing a stilbene moiety. The formula is $\mathrm{C}_{14} \mathrm{H}_{12} \mathrm{O}_{3}$. It is found in several plants, including grapes and blueberries (19). A previous clinical pharmacological study has demonstrated that the cell proliferation antigen Ki-60 expression in tumor tissues is significantly decreases after patients are treated with Res, which means that Res may have potential antitumor effects (20). The antitumor mechanism of Res is mainly through cell cycle arrest and the induction of apoptosis, including upregulation of p21 cyclin-dependent kinase inhibitor 1 (Cip1/WAF1), Bax/Bcl-2, Bak and p53 and activation of caspases signaling pathways (21). Several in vitro and in vivo studies have shown that Res inhibits the proliferation of several cancer types, including breast cancer, leukemia, colon cancer, lung cancer, liver cancer and thyroid cancer $(22,23)$. In addition, studies have shown that resveratrol inhibits cell proliferation by interfering with AR synthesis in prostate cancer cells $(24,25)$.

The present study investigated the effects of Res in vitro on proliferation and apoptosis in PCa cell lines (LNCaP and LNCaP-B). Alterations in the expression of AR protein and the levels of AKT phosphorylation were analyzed. Finally, the effects of Res on PCa cell proliferation and apoptosis via ARV7 and the PI3K/AKT signaling pathway were measured.

\section{Materials and methods}

Cell culture. The human PCa cell line LNCaP and human normal prostate cell line RWPE-1 were obtained from Shanghai Institute of Biochemistry and Cell Biology. Both cell lines purchased are certified by the American Type Culture Collection. PCa cells were routinely cultured in RPMI-1640 medium supplemented with $10 \%$ (v/v) fetal bovine serum (FBS) and RWPE-1 cells were cultured according to the manufacture's direction in K-SFM medium (all Gibco; Thermo Fisher Scientific, Inc.), penicillin $100 \mathrm{U} / \mathrm{ml}$ and streptomycin $100 \mathrm{U} / \mathrm{ml}$ (both Beijing Solarbio Science \& Technology Co., Ltd.) at $37^{\circ} \mathrm{C}$ under an atmosphere of $5 \% \mathrm{CO}_{2}$ in humidified air.

Reagents and antibodies. Resveratrol (Res), docetaxel (PC) and bicalutamide were purchased from Sigma-Aldrich; Merck KGaA and were dissolved in $100 \%$ DMSO to create stock solutions of $50 \mathrm{mM}$ and stored at $-20^{\circ} \mathrm{C}$. These were subsequently diluted in RPMI-1640 medium to the desired concentration for the experiments and the final concentration of DMSO that was given to the cells was $0.05 \%$. Antibodies against AKT (cat. no. 4685), phosphorylated (p-)AKT (cat. no. 4058) were obtained from Cell Signaling Technology, Inc. Antibodies against AR (cat. no. 133273), ARV7 (cat. no. 15656) and GAPDH (cat. no. 9485) were purchased from Abcam. GAPDH was used as a loading control. The goat anti-rabbit and anti-mouse IgG-HRP secondary antibodies were purchased from Wuhan Boster Biological Technology, Ltd. The AKT pathway activator (phen) was purchased from Selleck Chemicals, primers (ARV7 and GAPDH) were purchased from Angen Biotech Co., Ltd. SYBR ${ }^{\circledR}$ Premix Ex Taq ${ }^{\mathrm{TM}}$ II (Tli RNaseH Plus) and the PrimeScript ${ }^{\mathrm{TM}}$ RT Reagent kit with gDNA Eraser (Perfect Real Time) were purchased from Bao Biological Engineering Co., Ltd. (http://www.takara. com.cn). Lipofectamine ${ }^{\circledR} 2000$ was purchased from Thermo Fisher Scientific, Inc., and the miRcute miRNA First-Strand cDNA Synthesis kit was purchased from Tiangen Biochemical Technology Co., Ltd.

Construction of the hormone-resistant PCacell line (LNCaP-B) and induction of ARV7 over-expression in LNCAP-B cells. $\mathrm{LNCaP}$ cells in the androgen-dependent logarithmic growth phase were digested with trypsin and inoculated in a medium containing $10 \%$ non-androgen (activated charcoal-treated) FBS and cultured at $37^{\circ} \mathrm{C}$ with $5 \% \mathrm{CO}_{2}$. After 10 generations of culture, bicalutamide $(1.0 \mu \mathrm{M})$ was added to the culture medium for 20 generations, and then the concentration of bicalutamide was increased to $5.0 \mu \mathrm{M}$ to continue subculture. By 6 months of subculture, hormone-resistant prostate cancer cells had been constructed successfully.

The ARV7 overexpression vector was based on the PCDNA3.1 plasmid (Guangzhou RiboBio Co., Ltd.). The primers were designed and the RNA fragment of the ARV7 gene was obtained using the cell genome cDNA as a template. The fragment was inserted into multiple cloning sites downstream of the plasmid PCDNA3.1 to obtain the wild-type ARV7-overexpression vector. The PCDNA3.1-ARV7 plasmid and DH5 $\alpha$ competent cells mixed culture PCDNA3.1-ARV7. The constructed PCDNA3.1-ARV7 vector and PCDNA3.1 vector were introduced into LNCaP-B cells, respectively, using Lipofectamine 2000 reagent according to the manufacturer's instructions, and ARV7 protein expression was verified using western blotting.

Cell viability assay. Cell viability was determined using an MTT assay. Briefly, the PCa cell lines LNCaP and LNCaP-B $\left(5 \times 10^{3}\right.$ cells/well) were seeded in 96 -well plates. Cells were cultured overnight $\left(37^{\circ} \mathrm{C}, 5 \% \mathrm{CO}_{2}\right)$, and the cells were incubated with various concentrations of $\operatorname{RES}(25,50,100$ and $200 \mu \mathrm{M})$ dissolved in DMSO and PC $(5 \mu \mathrm{M})$ and various concentrations (200 $\mu \mathrm{l} /$ wells) of RES, RES+ARV7 and RES+phen for $12,24,48$ and $72 \mathrm{~h}$ (at $37^{\circ} \mathrm{C}$ in $5 \% \mathrm{CO}_{2}$ ). DMSO (0.05\%) was used as the negative control (NC) group. MTT $(0.5 \mathrm{mg} / \mathrm{ml}$, $20 \mu \mathrm{l} /$ well) was added to each well $4 \mathrm{~h}$ in advance. After $4 \mathrm{~h}$ incubation (at $37^{\circ} \mathrm{C}$ in $5 \% \mathrm{CO}_{2}$ ), DMSO (150 $\mu \mathrm{l} /$ well) was added to each well, and the cells were measured at $492 \mathrm{~nm}$ using a Multiskan Ascent microplate photometer. Viability was expressed as the percent viable cells compared with vehicle-treated control cells that were arbitrarily assigned as $100 \%$ viability.

Flow cytometric analysis for apoptosis assay. A flow cytometric assay was conducted to analyze the apoptosis of $\mathrm{PCa}$ cells. Cells plated in $6-\mathrm{cm}$ plates were treated with various concentrations of Res (50 and $100 \mu \mathrm{M}), \mathrm{PC}(5 \mu \mathrm{M}), \mathrm{RES}+\mathrm{ARV} 7$ and RES+phen. After 24-h treatment, cells were washed with phosphate buffer saline and harvested. The apoptosis assay was performed according to the manufacturer's protocol of 
the Annexin V/7-ADD apoptosis kit (BD Pharmingen ${ }^{\mathrm{TM}}$; BD Biosciences). The sedimented cells were resuspended with $500 \mathrm{ul}$ binding buffer based on the instructions. Subsequently, $5 \mu \mathrm{l}$ Annexin V-7AAD and $5 \mu \mathrm{l}$ Annexin V-APC were added to the cell suspension and cells were incubated at room temperature for $15 \mathrm{~min}$ in the dark. The stained cells were analyzed using BD FACSCanto II flow cytometry (BD Pharmingen ${ }^{\mathrm{TM}}$; BD Biosciences) and the data analyses were performed using SPSS 19.0 software (IBM, Corp.).

Reverse transcription-quantitative $(R T-q) P C R$. Total RNA was extracted from LNCaP and LNCaP-B cells treated with various drugs in each group, using TRIzol reagent (Invitrogen; Thermo Fisher Scientific, Inc.). The concentration and purity of RNA were measured using UV spectrophotometry (Thermo Fisher Scientific, Inc.). One microgram of the total RNA was used as a template and was reverse transcribed into cDNA using PrimeScript ${ }^{\mathrm{TM}}$ RT Reagent kit with gDNA Eraser (Perfect Real Time) to obtain a template of the reaction system. The condition for reverse transcription was at $37^{\circ} \mathrm{C}$ for $15 \mathrm{~min}$, followed by $85^{\circ} \mathrm{C}$ for $5 \mathrm{sec}$. The expression levels of AR, ARV7 and GAPDH in the cells were analyzed using two-step fluorescence quantitative PCR. A 10- $\mu$ l reaction system was generated. The qPCR reaction was performed using the LightCycler 480 II DNA Amplifier according to the manufacturer's instructions. The reaction conditions were as follows: Pre-denaturation at $95^{\circ} \mathrm{C}$ for $2 \mathrm{~min}$ followed by $95^{\circ} \mathrm{C}$ for $15 \mathrm{sec}$, and $60^{\circ} \mathrm{C}$ for $30 \mathrm{sec}$ was performed for a total of 40 cycles. After completion of the reaction, the relative expression levels of AR and ARV7 in the cells were expressed using $2^{-\Delta \Delta C q}$ normalization (26). The designed specific primers for each gene are listed in Table I.

Western blot analysis. PCa cells were treated with various concentrations of Res or PC for $24 \mathrm{~h}$. Cells were lysed in RIPA lysis buffer (Beyotime Institute of Biotechnology) and total proteins were extracted at $4^{\circ} \mathrm{C}$. The total protein concentrations were determined using the BCA assay (Beyotime Institute of Biotechnology). The proteins (20 $\mu \mathrm{g} /$ lane) were loaded on a $12 \%$ gel, and resolved using SDS-PAGE with a constant voltage of $80 \mathrm{~V}$. Then the proteins were transferred to PVDF membranes (EMD Millipore) using the Bio-Rad PowerPac Basic Power Supply system. Membranes were blocked in 5\% skimmed milk in Tris-Buffered Saline Tween-20, followed by incubation with primary antibodies overnight at $4^{\circ} \mathrm{C}$. The dilutions of ARV7, Bax, Bcl-2, AR, AKT, p-AKT and GAPDH were all 1:1,000. Blots were then probed with the IgG-HRP secondary antibody $(1: 1,000)$ at room temperature for $1 \mathrm{~h}$, and protein bands were detected using chemiluminescence reagent (EMD Millipore), under the Tanon5200 chemiluminescent imaging system (Tanon Science and Technology Co., Ltd.). ImageJ 1.8.0 software (National Institutes of Health) was used to quantify western blotting images.

Statistical analysis. All the data analyses were performed using SPSS 19.0 software. All experiments were repeated at least three times and $\mathrm{P}<0.05$ was considered to indicate a statistically significant difference. All experiments were performed in triplicate. Data are expressed as the mean \pm SD. A Student's unpaired t-test was used for determining the
Table I. The primer sequences of the reverse transcription quantitative-PCR.

\begin{tabular}{ll}
\hline Gene & \multicolumn{1}{c}{ Primer sequence $\left(5^{\prime} \rightarrow 3^{\prime}\right)$} \\
\hline$A R$ & F: AGGGCAGATCTTGTCCACCG \\
& R: TTGCTCAGAAGAGTTCAACA \\
$A R V 7$ & F: AGGGCAGATCTTGTCCACCG \\
& R: TTGCTCAGAAGAGTTCAACA \\
GAPDH & F: CGGAGTCAACGGATTTGGTCGTAT \\
& R: AGCCTTCTCCATGGTGGTGAAGAC
\end{tabular}

F, forward; R, reverse; AR, androgen-androgen receptor; ARV7, androgen-androgen receptor splice variant 7 .

differences between two groups and for more than two group comparisons one-way ANOVA test with a Tukey's post hoc test was used.

\section{Results}

Effects of Res on proliferation of PCa cells and effects of ARV7 overexpression on proliferation of LNCAP-B cells. Various concentrations of Res $(25,50,100$ and $200 \mu \mathrm{M})$ and PC $(5 \mu \mathrm{M})$ were used to treat LNCaP and LNCaP-B cells for 24,48 and $72 \mathrm{~h}$. The proliferative capacities of both cell types were significantly inhibited compared with the control, and the effects were time- and dose-dependent (Fig. 1A and B). The results suggested that Res inhibited the proliferation of PCa cells. Nevertheless, following administration of relatively various concentrations of $\operatorname{Res}(25,50,100$ and $200 \mu \mathrm{M})$ to RWPE-1 cells for $12,24,48$ and $72 \mathrm{~h}$, the viability of PCa cells was significantly lower compared with that of normal cells after Res treatment after 48 and $72 \mathrm{~h}$ (Fig. 1C). To further investigate the molecular mechanism of ARV7 inhibition in PCa cells mediated by Res, an overexpression vector (PCDNA3.1-ARV7) of ARV7 was constructed and transfected it into LNCaP-B cells. Western blot analysis verified that, compared with cells transfected with empty vector (PCDNA3.1), ARV7 protein levels in cells transfected with ARV7 overexpression vector (ARV7) increased significantly (Fig. 1D). It was demonstrated that the Res+ARV7 group and the Res+phen group restored the inhibitory effect of Res on the proliferation of LNCaP-B cells compared with Res treatment alone (Fig. 1E). This suggests that effect of Res on the proliferation of LNCaP-B cells may be associated with the ARV7 and AKT pathways.

Effects of Res on the apoptosis of PCa cells and the effect of ARV7 on apoptosis of LNCAP-B cells induced by Res. Flow cytometry was used to measure the effects of various concentrations of Res on apoptosis in LNCaP and LNCaP-B. Compared with the NC group, after treatment of LNCaP and LNCaP-B cells with various concentrations of Res $(50$ and $100 \mu \mathrm{M})$ for $24 \mathrm{~h}$, the apoptosis level of LNCaP cells increased by approximately 33.3 and $75.9 \%$, respectively, and the apoptosis level of LNCaP-B cells increased by approximately 9.6 and $33 \%$, respectively. (Q1, Q2, Q3, Q4 represented dead cells, late 
A

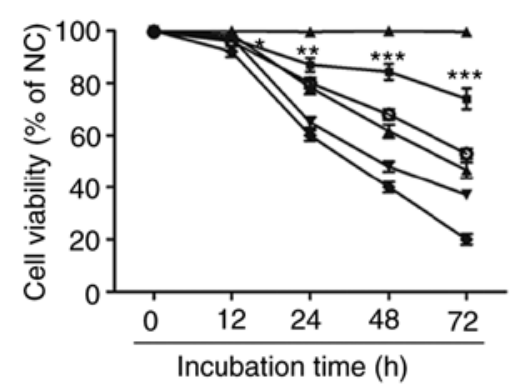

C

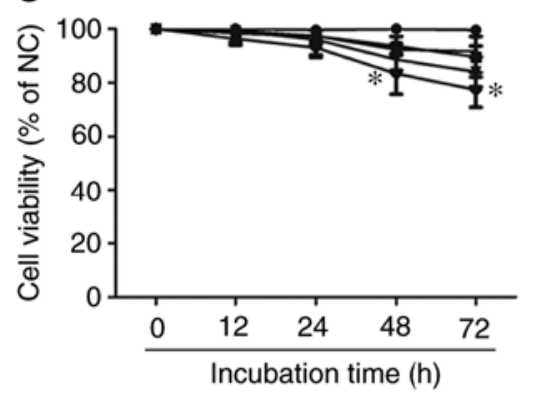

B

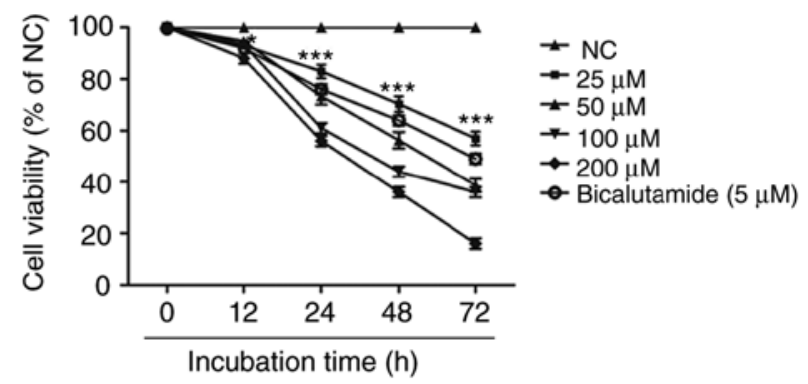

D

$\rightarrow$ NC

$-25 \mu \mathrm{M}$

$\mp 50 \mu \mathrm{M}$

$-100 \mu \mathrm{M}$

$\rightarrow 200 \mu \mathrm{M}$
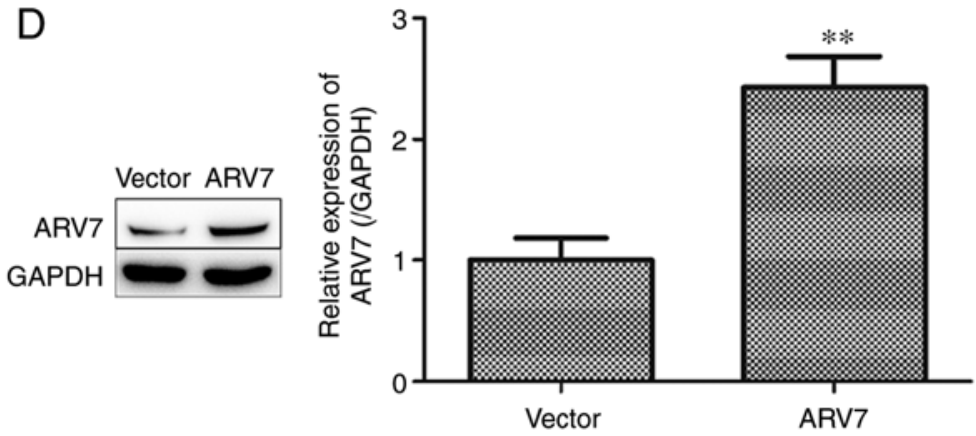

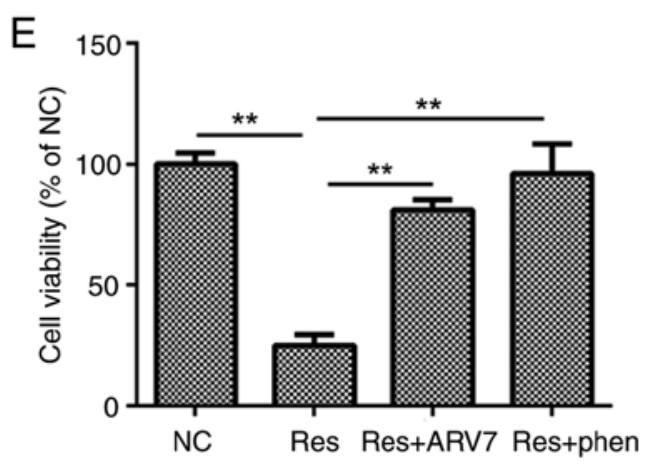

Figure 1. Res inhibits LNCaP and LNCaP-B cell proliferation and the effects of ARV7 overexpression on proliferation of LNCaP-B cells. (A and B) The cell viability of LNCaP and LNCaP-B cells exhibited a significant decrease in a dose- and time-dependent manner, which was measured using an MTT assay. (C) The effect of Res on the cell viability of RWPE-1 cells was significantly less compared with PCa cells. (D) Western blot analysis verified that, compared with cells transfected with empty vector, ARV7 protein levels in cells transfected with the ARV7-overexpression vector increased significantly. (E) Compared with the Res+ARV7 group and the Res+phen group, Res group shows stronger inhibition in LNCaP-B cells. The data are presented as the mean \pm SD for three independent experiments, ${ }^{*} \mathrm{P}<0.05,{ }^{* *} \mathrm{P}<0.01$ and ${ }^{* * * *} \mathrm{P}<0.001$, vs. cells treated with DMSO (control). Res, resveratrol; ARV7, AR splicing variant 7; Vector, empty vector; phen; AKT pathway activator.

apoptotic cells, viable cells, and early apoptotic cells, respectively.) Therefore, the total number of early and late apoptotic cells in the two cell lines was significantly higher compared with those in the control group. The effect of Res on apoptosis of LNCaP and LNCaP-B cells was concentration-dependent $(\mathrm{P}<0.05$ and $\mathrm{P}<0.01$; Fig. $2 \mathrm{~A}$ and $\mathrm{B}$, respectively).

The effects of overexpression of ARV7 on Res-induced apoptosis in LNCaP-B cells were also studied. Upregulation of ARV7 suppressed Res-induced apoptosis by approximately $26 \%$. It was reported that Res+ARV7 and Res+phen treatment reversed Res-induced apoptosis in LNCaP-B cells (Fig. 2C). In order to further study the relevant molecular mechanisms, the levels of apoptosis-associated proteins were measured using western blotting. The results showed that all concentrations of Res increased the expression levels of pro-apoptotic proteins Bax and the expression levels of antiapoptotic protein $\mathrm{Bcl} 2$ were lower compared with those in control group (Fig. 2D and E). In addition, compared with the effect of Res on PCa cells,
Res+ARV7 and Res+phen treatment increased the expression of antiapoptotic protein $\mathrm{Bcl} 2$ and decreased the expression of pro-apoptotic protein Bax (Fig. 2F). These results suggested that Res not only inhibits the proliferation of PCa cells, but also induces apoptosis. These results also suggested that the pro-apoptotic effect of Res on LNCaP-B cells is associated with the ARV7 and AKT pathway signaling pathways.

Effect of Res on secretion of PSA by PCa cells. Chemiluminescence detection showed that various concentrations of Res $(25,50$ and $100 \mu \mathrm{M})$ and PC (5 $\mu \mathrm{M})$ applied to LNCaP and LNCaP-B cells for $24 \mathrm{~h}$, caused PSA content in both cell lines to be significantly lower compared with that of the control group $\left({ }^{*} \mathrm{P}<0.05\right.$ and $\left.{ }^{* *} \mathrm{P}<0.01\right)$. Furthermore, it was also revealed that Res decreased PSA levels in LNCaP and LNCaP-B cells in a concentration-dependent manner (Fig. 3). This suggested that Res inhibited the secretion of PSA by LNCaP and LNCaP-B cells. 
A

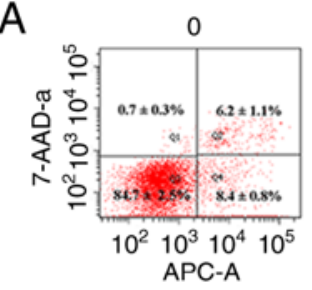

B

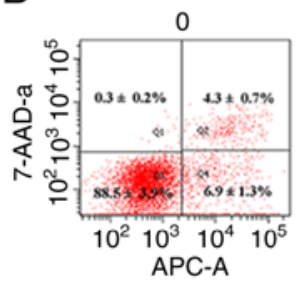

C

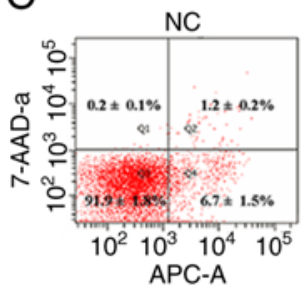

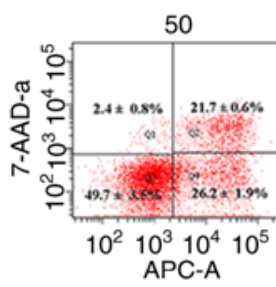
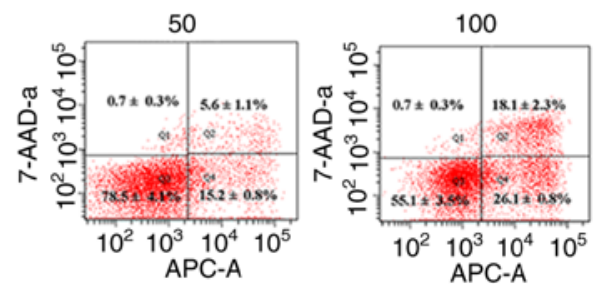

APC-A
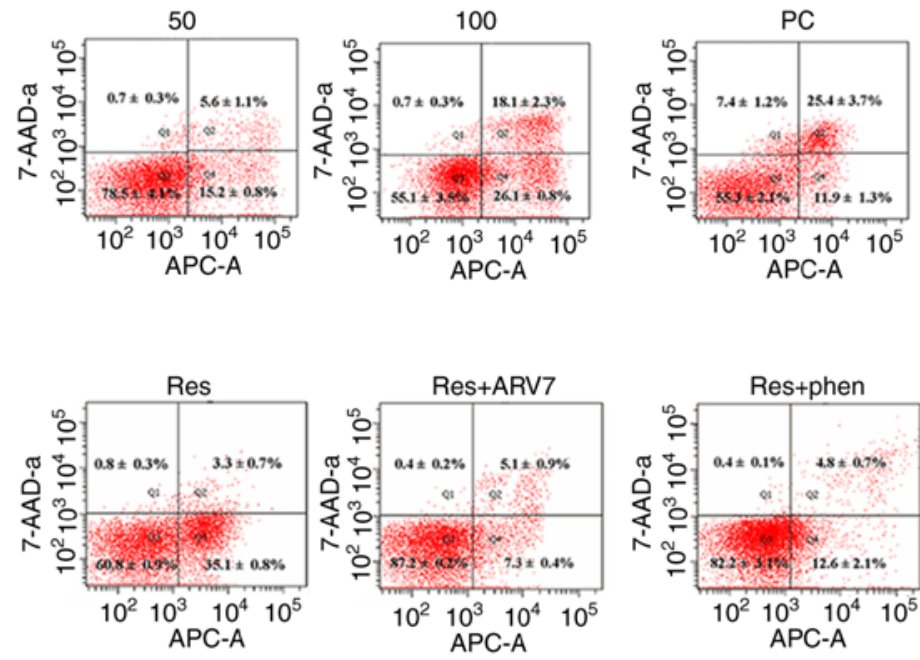

APC-A
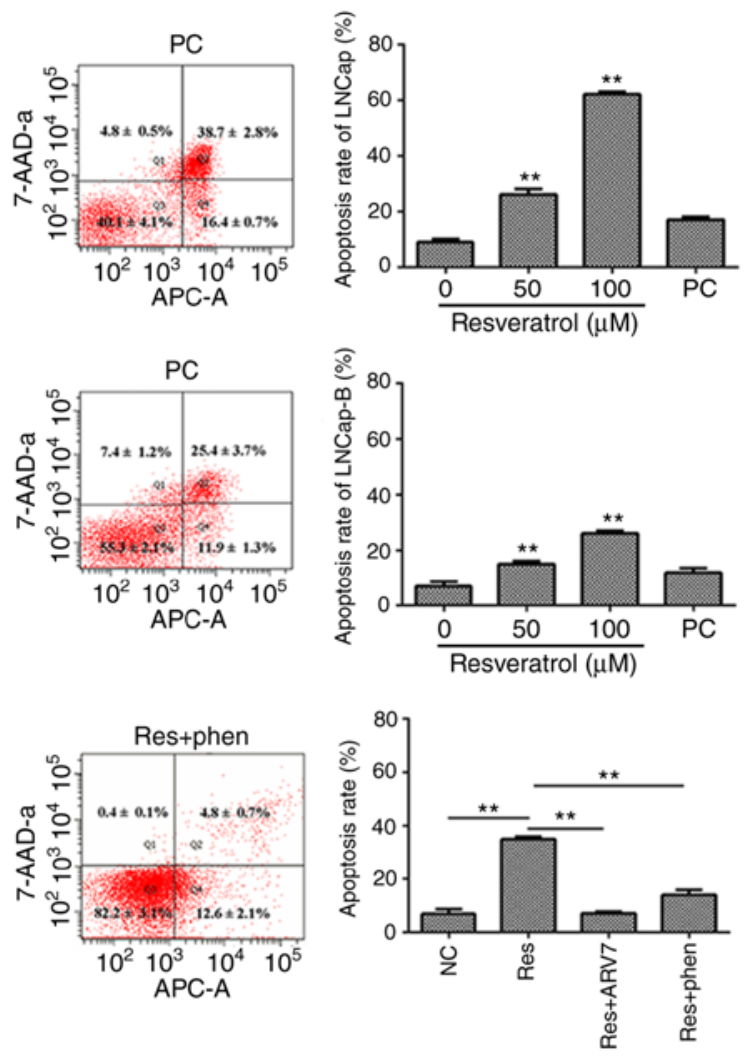

E

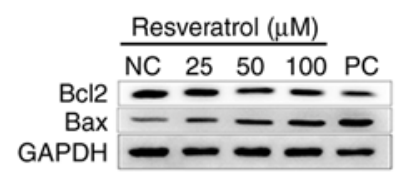

F

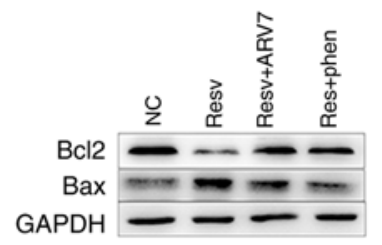

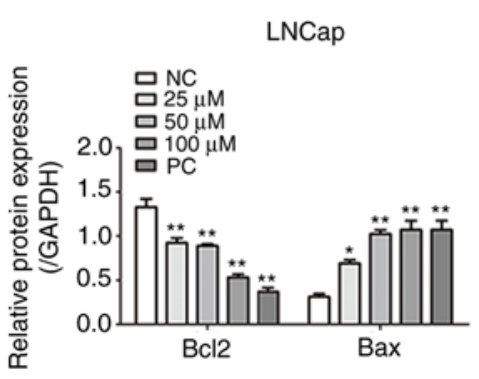
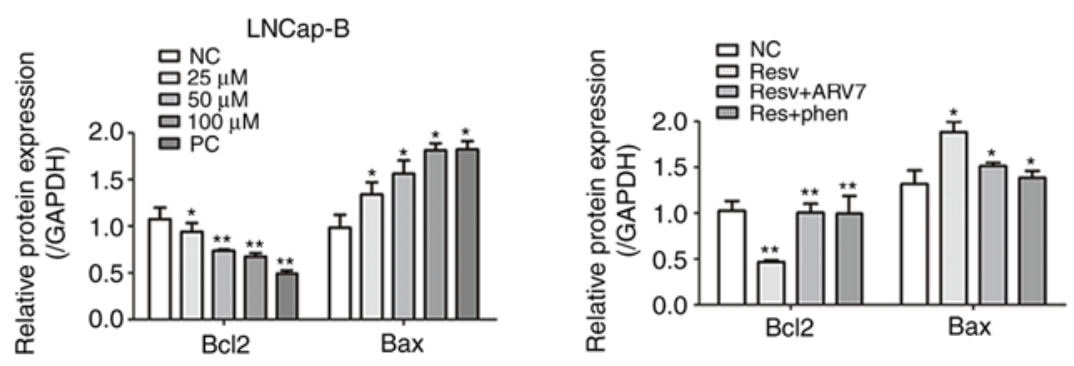

Figure 2. Res induces apoptosis in LNCaP and LNCaP-B cells and the effect of ARV7 on apoptosis of LNCaP-B cells. (A and B) LNCaP and LNCaP-B cells were treated with various concentrations of Res $(50$ and $100 \mu \mathrm{M})$ and PC $(5 \mu \mathrm{M})$ for $24 \mathrm{~h}$, then the cells were harvested and analyzed using Annexin V/7-ADD double staining. The column represents the corresponding apoptosis rate. (C) The effect of overexpression of ARV7 on Res-induced apoptosis was compared with the Res+ARV7 and the Res+phen group using Annexin V/7-ADD double staining. The column represents the corresponding apoptosis rate. (D and E) Western blotting analysis was conducted to measure the expression levels of apoptosis-associated proteins in the LNCaP and LNCaP-B cells following treatment with different concentrations of Res $(25,50$ and $100 \mu \mathrm{M})$ and PC $(5 \mu \mathrm{M})$. (F) Western blotting analysis was conducted to measure the expression levels of apoptotic-associated protein in LNCaP-B cells comparing with the Res+ARV7 and the Res+phen group. The data are presented as the mean \pm SD for three independent experiments. ${ }^{*} \mathrm{P}<0.05,{ }^{* *} \mathrm{P}<0.01$ vs. cells treated with DMSO (control). Res, resveratrol; ARV7, AR splicing variant 7 ; Vector, empty vector; phen; AKT pathway activator; NC, negative control; PC, docetaxel.

Effect of Res on AR mRNA levels in PCa cells. The effect of various concentrations of Res on mRNA levels in PCa cells was measured using RT-qPCR. It was demonstrated that, after treatment of $\mathrm{LNCaP}$ and $\mathrm{LNCaP}-\mathrm{B}$ cells with various concentrations of Res $(25,50$ and $100 \mu \mathrm{M})$ and PC $(5 \mu \mathrm{M})$ for $24 \mathrm{~h}$, expression levels of $A R$ mRNA in the two PCa cells were significantly lower compared with those of the control group (Fig. 4A and B). It was also reported found that, with increasing
Res concentrations, the effect of Res on the downregulation of $A R$ mRNA levels in PCa cells was dose-dependent. These results indicated that Res inhibited the expression levels of $A R$ in LNCap and LNCap-B cells in a dose-dependent manner.

Effect of Res on AR and ARV7 protein levels in PCa cells. The effect of various concentrations of Res on levels of AR protein in PCa cells was measured using western blotting. Expression 


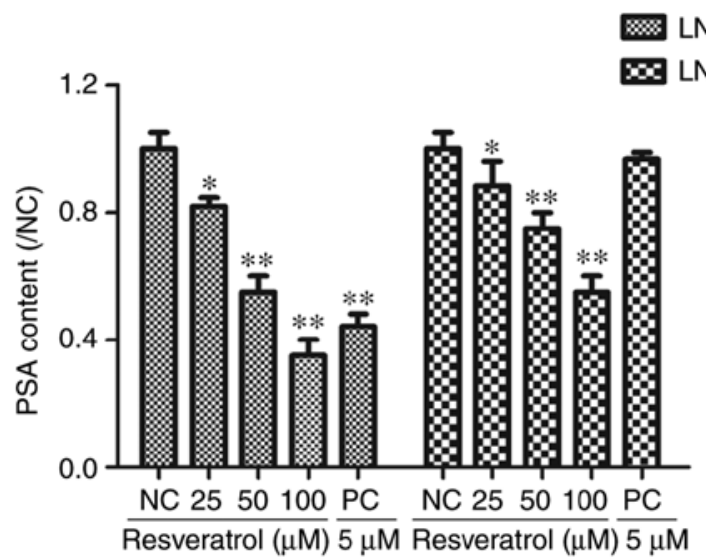

Figure 3. Chemiluminescence was conducted to measure PSA levels in LNCaP and LNCaP-B cells following treatment with different concentrations of Res $(25,50$ and $100 \mu \mathrm{M})$ and PC $(5 \mu \mathrm{M})$. The data are presented as the mean \pm SD for three independent experiments. ${ }^{*} \mathrm{P}<0.05$ and ${ }^{* *} \mathrm{P}<0.01$ vs. cells treated with DMSO (control). PSA, prostate specific antigen; Res, resveratrol; NC, negative control; PC, docetaxel.

levels of AR protein in the two PCa cells were significantly lower compared with those in the control group after treatment of LNCaP and LNCaP-B cells with various concentrations of Res $(25,50$ and $100 \mu \mathrm{M})$ for $24 \mathrm{~h}$. It was also shown that Res was associated with decreases of AR expression in PCa cells in a concentration-dependent manner (Fig. 5A and B). Western blot analysis showed that Res was associated with lower levels of AR protein in LNCaP and LNCaP-B cells.

Studies have shown that $\mathrm{PCa}$ castration-resistance is associated with expression levels of ARV7 $(27,28)$. Therefore, expression levels of ARV7 in LNCaP and LNCaP-B cells were examined using western blotting. ARV7 was expressed at low levels in LNCaP cells but positive in LNCaP-B (Fig. 5C). This suggested that the hormone resistance of LNCaP-B may be associated with high expression of ARV7. The effect of Res on ARV7 expression was further examined and it was revealed that Res inhibited ARV7 expression (Fig. 5D). This suggested that the inhibitory effect of Res on hormone-resistant LNCaP-B cells may be associated with the inhibition of ARV7 expression.

Effect of Res on the AKT signaling pathway in PCa cells and the effect of ARV7 on the AKT signaling pathway in LNCaP-B cells. Western blotting was used to measure the effect of Res on the AKT signaling pathway in PCa cells. After treatment of $\mathrm{LNCaP}$ and $\mathrm{LNCaP}-\mathrm{B}$ cells with various concentrations of Res $(25,50$ and $100 \mu \mathrm{M})$ for $24 \mathrm{~h}$, levels of p-AKT in the two PCa cells were significantly lower compared with those in the control group (Fig. 6A and B). Res inhibited the AKT phosphorylation in a concentration-dependent manner. These results suggested that Res may have inhibited AKT phosphorylation in LNCaP and LNCaP-B cells.

The molecular mechanism of Res-mediated ARV7 inhibition in PCa was further investigated. Compared with the Res group, the Res+ARV7 and Res+AKT pathway activator groups showed significantly higher levels of p-AKT (Fig. 6C). These results suggested that overexpression of ARV7 significantly decreased Res-induced phosphorylation of AKT.

\section{Discussion}

Advanced PCa is very common (29). It is usually treated with castration combined with anti-androgen medications; however, after a certain period of treatment, castration-resistant PCa often develops. Studies have demonstrated that about $90 \%$ of patients with PCa eventually develop into castration-resistant PCa and the average survival time is only $16-18$ months $(30,31)$. For castration-resistant $\mathrm{PCa}$, despite the fact that comprehensive treatment with chemotherapy and other treatments can prolong progression-free survival (PFS), these treatments ultimately fail to effectively prevent the progression of the disease (32). Therefore, improved treatments for castration-resistant $\mathrm{PCa}$ need to be developed.

Res has a structure similar to diethylstilbestrol and possesses estrogenic activity; it is considered to be a natural phytoestrogen (33). Kuwajerwala et al (34) showed that Res inhibited the proliferation of PCa cells. Res is expected to become a drug for clinical treatment of PCa. The present study demonstrated that Res significantly inhibited the proliferation of LNCaP and LNCaP-B cells in a concentration range of 25-100 $\mu \mathrm{M}$. This effect had a dual dependence on time and dose. In addition, the number of early apoptotic cells and late apoptotic cells in the two cell lines increased significantly after Res was applied to LNCaP and LNCaP-B cells for $24 \mathrm{~h}$. Moreover, the effect of Res on apoptosis of LNCaP and LNCaP-B cells also increased in a concentration-dependent manner. These results suggested that Res inhibits proliferation in androgen-dependent PCa LNCaP cells and hormone-resistant PCa LNCaP-B cells, possibly achieved by inducing apoptosis in LNCaP and LNCaP-B cells.

The prostate is a target organ of androgens, which are the most important factors affecting the growth of PCa. Androgens bind to the AR and activate the AR signaling pathway, thereby regulating $\mathrm{PCa}$ cell proliferation and apoptosis (35). A previous study have shown that castration resistance in $\mathrm{PCa}$ involves abnormalities regarding the AR, for example the AR signaling pathway remains activated (36). In addition, another study have shown that the AR is highly expressed in castration-resistant $\mathrm{PCa}$ and there is amplification of AR mRNA (37). Therefore, antagonizing or blocking $A R$ activity remains key in castration treatment of PCa. PSA has been suggested as a molecular target for prostate cancer because it is not only active in prostate tissues, but also plays a key role in the signaling pathway of PCa (14). In the current study, the effects of various concentrations of Res on PSA secretion and AR expression in PCa LNCaP and LNCaP-B cells were examined, and on the levels of AKT phosphorylation in PCa cells. It was shown that various concentrations of Res significantly inhibited secretion of PSA by LNCaP and LNCaP-B cells, inhibited the expression of AR mRNA and protein, and inhibited AKT phosphorylation. This inhibition was concentration-dependent. These data suggested that AR expression changes and abnormal signaling pathways are important factors in the treatment of hormone-resistant $\mathrm{PCa}$ cells, as well as hormone-dependent $\mathrm{PCa}$, and the emergence of AR-Vs is one of the important AR changes.

A Previous study have shown that ARV7 mRNA and protein levels are significantly increased in 

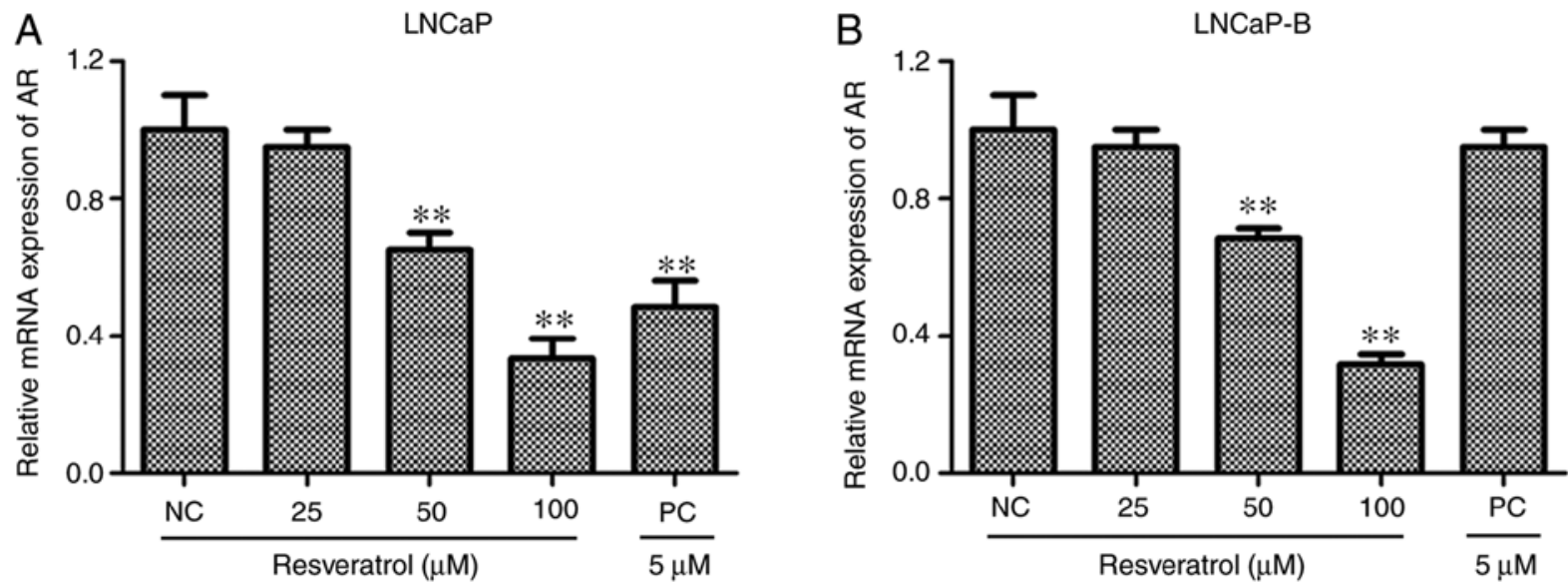

Figure 4. Reverse transcription-quantitative PCR was conducted to measure expression levels of AR mRNA in (A) LNCaP and (B) LNCaP-B cells that were treated with various concentrations of Res $(25,50$ and $100 \mu \mathrm{M})$ and PC $(5 \mu \mathrm{M})$ for $24 \mathrm{~h}$. The data are presented as the mean \pm SD for three independent experiments. ${ }^{* *} \mathrm{P}<0.01$ vs. cells treated with DMSO (control). AR, androgen-androgen receptor; Res, resveratrol; NC, negative control; PC, docetaxel.

A

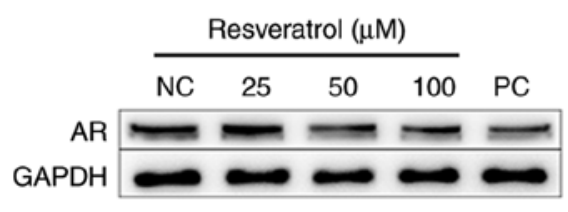

B

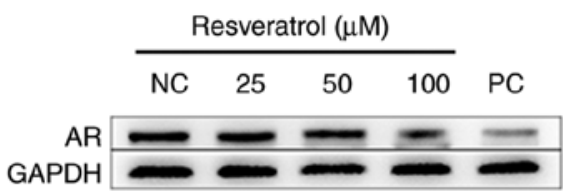

C

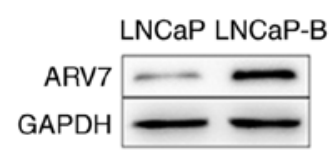

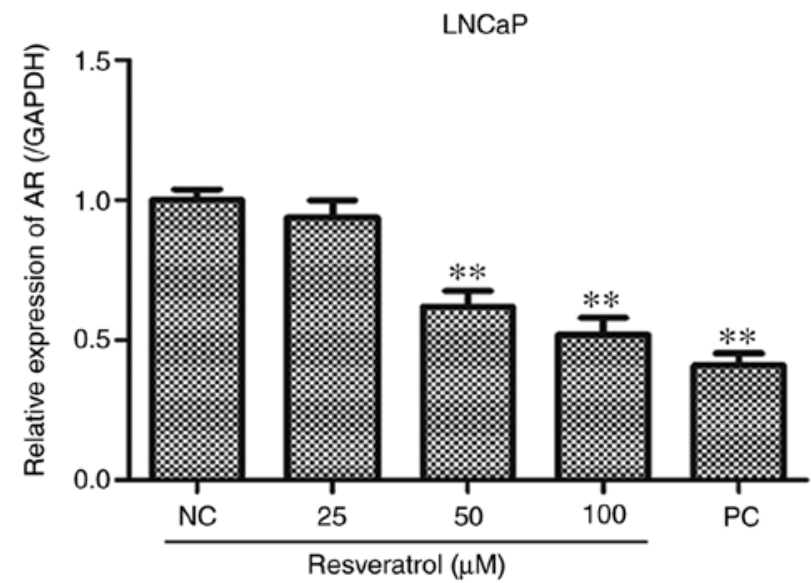

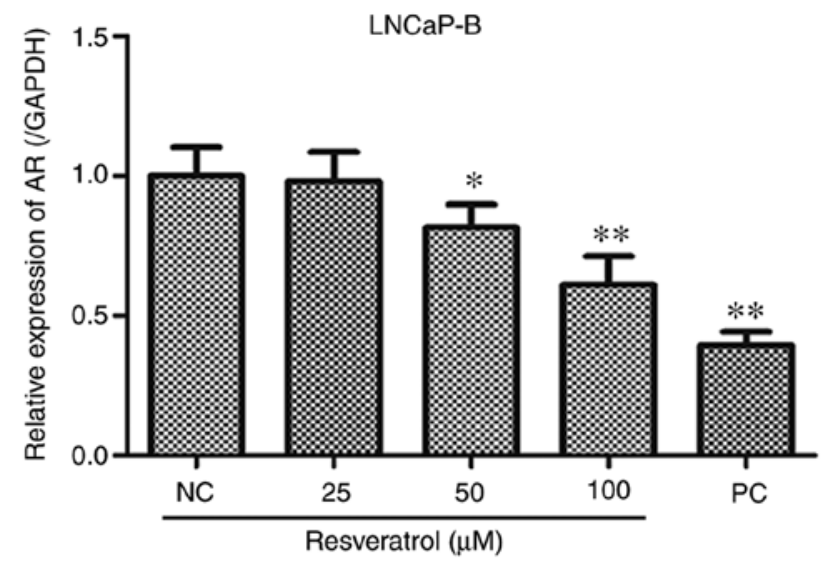

D

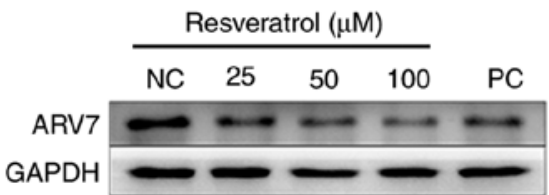

Figure 5. Res inhibits the expression of AR and ARV7 in LNCaP and LNCaP-B cells. (A and B) Western blotting analysis was conducted to measure the expression levels of AR protein in the LNCaP and LNCaP-B cells following treatment with different concentrations of Res $(25,50$ and $100 \mu \mathrm{M})$ and PC $(5 \mu \mathrm{M})$ for $24 \mathrm{~h}$. (C) Western blotting analysis was conducted to measure the expression levels of the AR splice variant ARV7 in in LNCaP and LNCaP-B cells. (D) Western blotting analysis was conducted to measure the expression levels of the AR splice variant ARV7 in the hormone resistance of LNCaP-B cells, which were treated with various concentrations of Res $(25,50$ and $100 \mu \mathrm{M})$ and PC $(5 \mu \mathrm{M})$ for $24 \mathrm{~h}$. The data are presented as the mean \pm SD for three independent experiments. ${ }^{*} \mathrm{P}<0.05,{ }^{* *} \mathrm{P}<0.01$ compared with the cells treated with DMSO (control). AR, androgen-androgen receptor; Res, resveratrol; NC, negative control; PC, docetaxel; ARV7, AR splicing variant 7. 
A

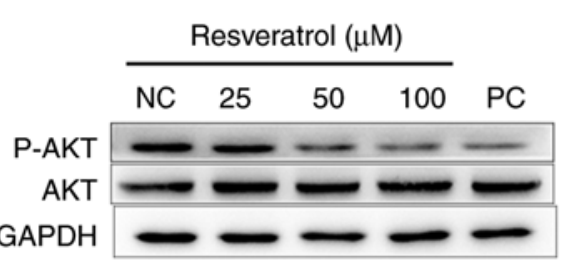

LNCap

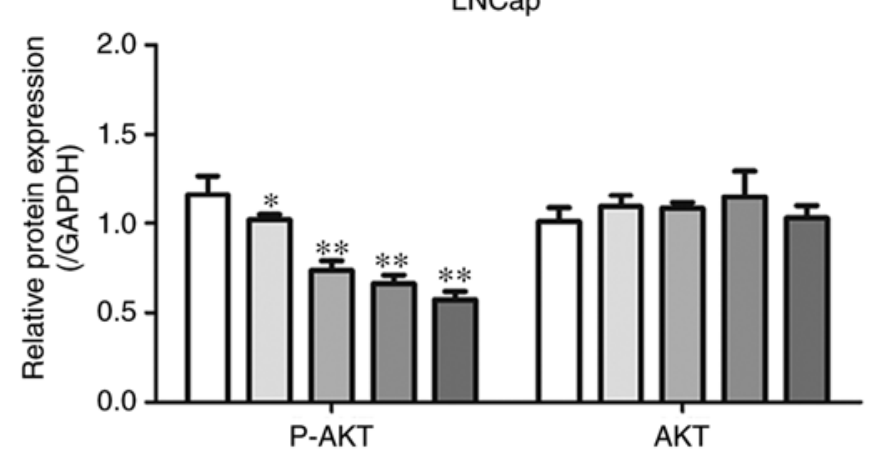

LNCap-B

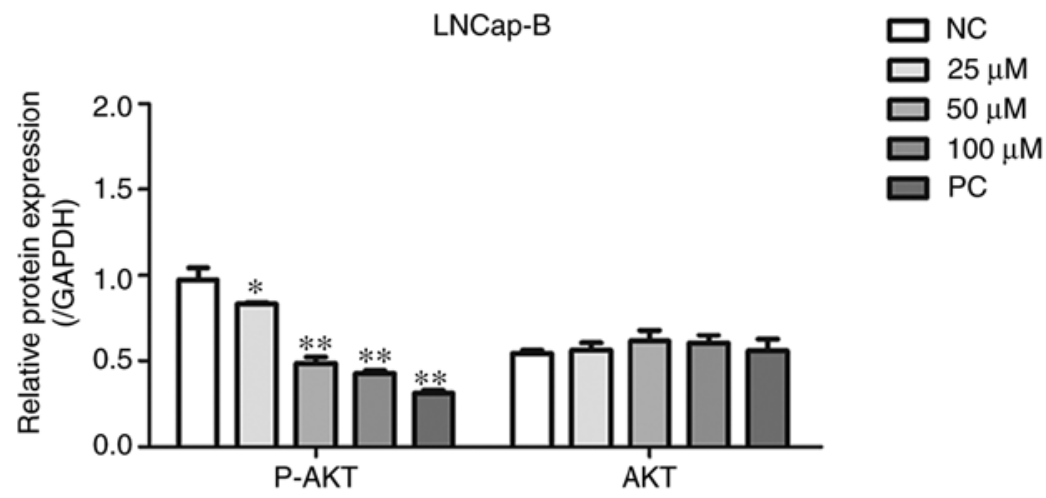

$\square \mathrm{NC}$

$\square 25 \mu \mathrm{M}$

$\square 50 \mu \mathrm{M}$

$\square 100 \mu \mathrm{M}$

$\square$ PC
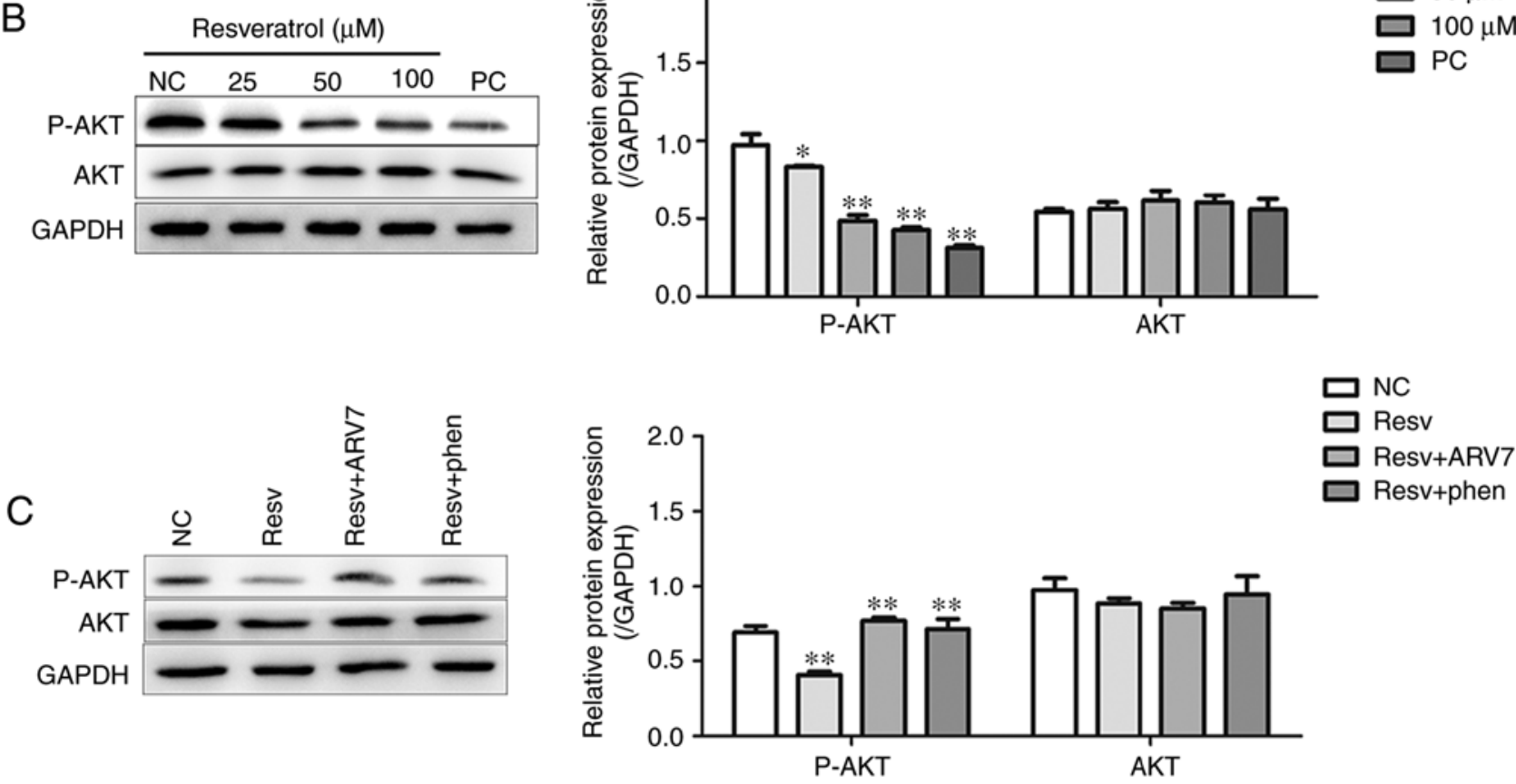

Figure 6. Res inhibits AKT phosphorylation in LNCaP and LNCaP-B cells. (A and B) Western blotting analysis was conducted to measure the expression levels of AKT signaling pathway protein in the $\mathrm{LNCaP}$ and $\mathrm{LNCaP}-\mathrm{B}$ cells following treatment with different concentrations of Res $(25,50$ and $100 \mu \mathrm{M})$ and PC $(5 \mu \mathrm{M})$. (C) Western blotting analysis was conducted to measure the expression levels of AKT protein in LNCaP-B cells compared with the Res+ARV7 and the Res+phen group. The data are presented as the mean \pm standard deviation for three independent experiments. ${ }^{*} \mathrm{P}<0.05$ and ${ }^{* *} \mathrm{P}<0.01$ vs. cells treated with DMSO (control). p-, phosphorylated; Res, resveratrol; NC, negative control; PC, docetaxel.

hormone-independent PCa cells and clinical specimens, and are associated with postoperative recurrence, distant metastasis and shortened survival time (38). It is also hypothesized that the structural features of AR-cleavage variants may activate downstream target genes through androgen-independent pathways to effect proliferation of $\mathrm{PCa}$ and eventually develop into androgen-independent PCa (39). This suggests that ARV7 may play an important role in castration-resistant $\mathrm{PCa}$. Moreover, it present study reported that ARV7 expression was low in LNCaP cells and higher in LNCaP-B cells, suggesting that the hormone resistance of LNCaP-B cells may be associated with high expression of ARV7. This result was consistent with the results of Guo et al (27), who suggested that ARV7 played an important role in castration-resistant PCa. The current study further examined the effect of Res on ARV7 expression and showed that Res inhibited ARV7 expression. It was also further demonstrated that inhibition of LNCaP-B cells by Res may be associated with the inhibition of ARV7 expression.

A previous review suggested that the PI3K/AKT signaling pathway is an important pathway affecting biological processes, including cell proliferation, cycle progression, migration and angiogenesis (40). Liao et al (41) showed that the intensity of AKT expression in prostate tumor tissues is positively correlated with PSA levels, and that increased PSA expression is associated with tumor progression. Another study showed that the activation of the PI3K/AKT pathway promotes the anti-apoptotic effect of PCa cells and the tumor progression (42). AKT is frequently activated in PCa cells, providing proliferation and survival signals, and regulating AR activity through phosphorylation (43). In line with these studies, the present results suggested 
that Res inhibits the AKT phosphorylation in $\mathrm{LNCaP}$ and LNCaP-B cells, and that the regulation of AR expression has a significant effect on hormone resistance. Res+ARV7 treatment elevated the levels of p-AKT, suggesting that overexpression of ARV7 reversed the inhibition effect of Res on AKT phosphorylation.in PCa. The inhibitory effect of Res on the AKT pathway is associated with ARV7. The effect of overexpression of ARV7 on Res inhibition of PCa proliferation and apoptosis was also investigated. Compared with the Res group, the Res+ARV7 and Res+phen groups restored the inhibitory effect of Res on LNCaP-B cell proliferation. It was shown that Res inhibited the proliferation of LNCaP-B cells in association with the ARV7 and AKT pathways. The apoptosis assay showed that the Res+ARV7 and Res+phen groups restored the proapoptotic effect of Res in LNCaP-B cells, and that this effect was associated with the ARV7 and AKT pathways.

Taken together, the present data suggested that Res inhibits the proliferative capacity of androgen-dependent LNCaP cells and hormone-resistant LNCaP-B cells, and induced apoptosis in both of these PCa cell lines. Res also inhibited the secretion of PSA. These effects may be associated with Res inhibiting AKT phosphorylation in $\mathrm{LNCaP}$ and $\mathrm{LNCaP}-\mathrm{B}$ cells and by regulation of AR mRNA and protein expression levels. It was also demonstrated that Res induced proliferation and apoptosis in LNCaP-B cells, possibly by causing decreased expression of ARV7 and inhibiting the activation of the AKT pathway. One limitation of the present study is that it was limited to in vitro data. Therefore, in the future, in vivo studies should be considered. In summary, these findings show the value of investigating the anticancer effects of Res and may provide a preliminary theoretical basis for its clinical application in the treatment of PCa.

\section{Acknowledgements}

Not applicable.

\section{Funding}

The present study was supported by the National Natural Science Funds of China (grant no. 81272833).

\section{Availability of data and materials}

All data generated or analyzed during this study were included in this published article.

\section{Authors' contributions}

MSY and JJL designed the experiments. MSY, HST, ZL, XJC and SHL performed the experiments. MSY, JRM and JJL were involved in data collection and statistical analyses. MSY, SHL, JRM and JJL wrote the article and prepared figures. JJL provided guidance and the financial support. All authors read and approved the final manuscript.

\section{Ethics approval and consent to participate}

Not applicable.

\section{Patient consent for publication}

Not applicable.

\section{Competing interests}

The authors declare that they have no competing interests.

\section{References}

1. Siegel RL, Miller KD and Jemal A: Cancer statistics, 2018. CA Cancer J Clin 68: 7-30, 2018.

2. Park JW, Jang WS, Koh DH, Ham WS, Rha KH, Hong SJ and Choi YD: Impact of early salvage androgen deprivation therapy in localized prostate cancer after radical prostatectomy: A propensity score matched analysis. Yonsei Med J 59: 580-587, 2018.

3. Sharifi N, Gulley JL and Dahut WL: Androgen deprivation therapy for prostate cancer. JAMA 294: 238-244, 2005.

4. Scher HI, Beer TM, Higano CS, Anand A, Taplin ME, Efstathiou E, Rathkopf D, Shelkey J, Yu EY, Alumkal J, et al: Antitumour activity of MDV3100 in castration-resistant prostate cancer: A phase 1-2 study. Lancet 375: 1437-1446, 2010.

5. Taneja SS: Re: Abiraterone in metastatic prostate cancer without previous chemotherapy. J Urol 190: 880, 2013.

6. Shafi AA, Yen AE and Weigel NL: Androgen receptors in hormone-dependent and castration-resistant prostate cancer. Pharmacol Ther 140: 223-238, 2013.

7. Ryan CJ and Tindall DJ: Androgen receptor rediscovered: The new biology and targeting the androgen receptor therapeutically. J Clin Oncol 29: 3651-3658, 2011.

8. Tilley WD, Buchanan G, Hickey TE and Bentel JM: Mutations in the androgen receptor gene are associated with progression of human prostate cancer to androgen independence. Clin Cancer Res 2: 277-285, 1996.

9. He B, Gampe RJ Jr, Kole AJ, Hnat AT, Stanley TB, An G, Stewart EL, Kalman RI, Minges JT and Wilson EM: Structural basis for androgen receptor interdomain and coactivator interactions suggests a transition in nuclear receptor activation function dominance. Mol Cell 16: 425-438, 2004.

10. Shang Y, Myers $M$ and Brown M: Formation of the androgen receptor transcription complex. Mol Cell 9: 601-610, 2002.

11. Montgomery RB, Mostaghel EA, Vessella R, Hess DL, Kalhorn TF, Higano CS, True LD and Nelson PS: Maintenance of intratumoral androgens in metastatic prostate cancer: A mechanism for castration-resistant tumor growth. Cancer Res 68: 4447-4454, 2008.

12. Waltering KK, Helenius MA, Sahu B, Manni V, Linja MJ, Jänne OA and Visakorpi T: Increased expression of androgen receptor sensitizes prostate cancer cells to low levels of androgens. Cancer Res 69: 8141-8149, 2009.

13. Chen CD, Welsbie DS, Tran C, Baek SH, Chen R, Vessella R, Rosenfeld MG and Sawyers CL: Molecular determinants of resistance to antiandrogen therapy. Nat Med 10: 33-39, 2004.

14. Moradi A, Srinivasan S, Clements J and Batra J: Beyond the biomarker role: Prostate-specific antigen (PSA) in the prostate cancer microenvironment. Cancer Metastasis Rev 38: 333-346, 2019.

15. Reebye V, Querol Cano L, Lavery DN, Brooke GN, Powell SM, Chotai D, Walker MM, Whitaker HC, Wait R, Hurst HC and Bevan CL: Role of the HSP90-associated cochaperone p23 in enhancing activity of the androgen receptor and significance for prostate cancer. Mol Endocrinol 26: 1694-1706, 2012.

16. Lin CY, Jan YJ, Kuo LK, Wang BJ, Huo C, Jiang SS, Chen SC, Kuo YY, Chang CR and Chuu CP: Elevation of androgen receptor promotes prostate cancer metastasis by induction of epithelial-mesenchymal transition and reduction of KAT5. Cancer Sci 109: 3564-3574, 2018.

17. Hu R, Dunn TA, Wei S, Isharwal S, Veltri RW, Humphreys E, Han M, Partin AW, Vessella RL, Isaacs WB, et al: Ligand-independent androgen receptor variants derived from splicing of cryptic exons signify hormone-refractory prostate cancer. Cancer Res 69: 16-22, 2009.

18. Hörnberg E, Ylitalo EB, Crnalic S, Antti H, Stattin P, Widmark A, Bergh A and Wikström P: Expression of androgen receptor splice variants in prostate cancer bone metastases is associated with castration-resistance and short survival. PLoS One 6: e19059, 2011.

19. Stewart JR, Artime MC and O'Brian CA: Resveratrol: A candidate nutritional substance for prostate cancer prevention. J Nutr 133 (7 Suppl): 2440S-2443S, 2003. 
20. Patel KR, Brown VA, Jones DJ, Britton RG, Hemingway D, Miller AS, West KP, Booth TD, Perloff M, Crowell JA, et al: Clinical pharmacology of resveratrol and its metabolites in colorectal cancer patients. Cancer Res 70: 7392-7399, 2010.

21. Aggarwal BB, Bhardwaj A, Aggarwal RS, Seeram NP, Shishodia S and Takada Y: Role of resveratrol in prevention and therapy of cancer: Preclinical and clinical studies. Anticancer Res 24: 2783-2840, 2004

22. Aluyen JK, Ton QN, Tran T, Yang AE, Gottlieb HB and Bellanger RA: Resveratrol: Potential as anticancer agent. J Diet (Suppl 9): 45-56, 2012.

23. Delmas D, Lancon A, Colin D, Jannin B and Latruffe $\mathrm{N}$ : Resveratrol as a chemopreventive agent: A promising molecule for fighting cancer. Curr Drug Targets 7: 423-442, 2006.

24. Jang YG, Go RE, Hwang KA and Choi KC: Resveratrol inhibits DHT-induced progression of prostate cancer cell line through interfering with the AR and CXCR4 pathway. J Steroid Biochem Mol Biol 192: 105406, 2019.

25. Mitchell SH, Zhu W and Young CY: Resveratrol inhibits the expression and function of the androgen receptor in $\mathrm{LNCaP}$ prostate cancer cells. Cancer Res 59: 5892-5895, 1999.

26. Livak KJ and Schmittgen TD: Analysis of relative gene expression data using real-time quantitative PCR and the 2(-Delta Delta $\mathrm{C}(\mathrm{T}))$ method. Methods 25: 402-408, 2001.

27. Guo Z, Yang X, Sun F, Jiang R, Linn DE, Chen H, Chen H, Kong X, Melamed J, Tepper CG, et al: A novel androgen receptor splice variant is up-regulated during prostate cancer progression and promotes androgen depletion-resistant growth. Cancer Res 69: 2305-2313, 2009.

28. Sobhani N, Generali D, D'Angelo A, Aieta M and Roviello G: Current status of androgen receptor-splice variant 7 inhibitor niclosamide in castrate-resistant prostate-cancer. Invest New Drug 36: 1133-1137, 2018.

29. Deng Y, Bi R, Zhu Z, Li S, Xu B, Rather WA and Wang C: A surveillance, epidemiology and end results database analysis of the prognostic value of organ-specific metastases in patients with advanced prostatic adenocarcinoma. Oncol Lett 18: 1057-1070, 2019.

30. Saad F, Chi KN, Finelli A, Hotte SJ, Izawa J, Kapoor A, Kassouf W, Loblaw A, North S, Rendon R, et al: The 2015 CUA-CUOG Guidelines for the management of castration-resistant prostate cancer (CRPC). Can Urol Assoc J 9: 90-96, 2015.

31. Penning TM: Mechanisms of drug resistance that target the androgen axis in castration resistant prostate cancer (CRPC). J Steroid Biochem Mol Biol 153: 105-113, 2015.

32. Thakur A, Vaishampayan U and Lum LG: Immunotherapy and immune evasion in prostate cancer. Cancers 5: 569-590, 2013.

33. Jang M, Cai L, Udeani GO, Slowing KV, Thomas CF, Beecher CW, Fong HH, Farnsworth NR, Kinghorn AD, Mehta RG, et al: Cancer chemopreventive activity of resveratrol, a natural product derived from grapes. Science 275: 218-220, 1997.
34. Kuwajerwala N, Cifuentes E, Gautam S, Menon M, Barrack ER and Reddy GP: Resveratrol induces prostate cancer cell entry into s phase and inhibits DNA synthesis. Cancer Res 62: 2488-2492, 2002.

35. Isaacs JT and Isaacs WB: Androgen receptor outwits prostate cancer drugs. Nat Med 10: 26-27, 2004

36. Scher HI and Sawyers CL: Biology of progressive, castration-resistant prostate cancer: Directed therapies targeting the androgen-receptor signaling axis. J Clin Oncol 23: 8253-8261, 2005.

37. Thakur MK, Heilbrun LK, Sheng S, Stein M, Liu G, Antonarakis ES, Vaishampayan U, Dzinic SH, Li X, Freeman S, et al: A phase II trial of ganetespib, a heat shock protein 90 Hsp90) inhibitor, in patients with docetaxel-pretreated metastatic castrate-resistant prostate cancer (CRPC)-a prostate cancer clinical trials consortium (PCCTC) study. Invest New Drugs 34: 112-118, 2005.

38. Vellky JE, Bauman TM, Ricke EA, Huang W and Ricke WA: Incidence of androgen receptor and androgen receptor variant 7 coexpression in prostate cancer. Prostate 79: 1811-1822, 2019.

39. Hsu CL, Liu JS, Wu PL, Guan HH, Chen YL, Lin AC, Ting HJ, Pang ST, Yeh SD, Ma WL, et al: Identification of a new androgen receptor (AR) co-regulator BUD31 and related peptides to suppress wild-type and mutated AR-mediated prostate cancer growth via peptide screening and X-ray structure analysis. Mol Oncol 8: 1575-1587, 2014.

40. Chen H, Zhou L, Wu X, Li R, Wen J, Sha J and Wen X: The PI3K/AKT pathway in the pathogenesis of prostate cancer. Front Biosci (Landmark Ed) 21: 1084-1091, 2016.

41. Liao Y, Grobholz R, Abel U, Trojan L, Michel MS, Angel P and Mayer D: Increase of AKT/PKB expression correlates with gleason pattern in human prostate cancer. Int J Cancer 107: 676-680, 2003

42. Pilling AB and Hwang C: Targeting prosurvival BCL2 signaling through Akt blockade sensitizes castration-resistant prostate cancer cells to enzalutamide. Prostate 79: 1347-1359, 2019.

43. Lin HK, Hu YC, Yang L, Altuwaijri S, Chen YT, Kang HY and Chang C: Suppression versus induction of androgen receptor functions by the phosphatidylinositol 3-kinase/Akt pathway in prostate cancer LNCaP cells with different passage numbers. J Biol Chem 278: 50902-50907, 2003.

This work is licensed under a Creative Commons Attribution-NonCommercial-NoDerivatives 4.0 International (CC BY-NC-ND 4.0) License. 